REVIEW

\title{
Innate lymphoid cell subsets and their cytokines in autoimmune diseases
}

\author{
Asma Maleki ${ }^{1}$, Jamal Motallebzadeh Khanmiri ${ }^{1}$, Mohammad Khani Eshrat Abadi ${ }^{1}$, \\ Faezeh Soveyzi ${ }^{2}$, Bobak Moazzami ${ }^{3,4}$, Michael R. Hamblin ${ }^{5,6}$, Nima Rezaei ${ }^{3,4,7}$ \\ ${ }^{1}$ Department of Hematology, School of Allied Medical Sciences, Tehran University of Medical Sciences (TUMS), Tehran, Iran \\ ${ }^{2}$ School of Medicine, Tehran University of Medical Sciences, Tehran, Iran \\ ${ }^{3}$ Research Center for Immunodeficiencies, Pediatrics Center of Excellence, Children's Medical Center, Tehran University of Medical Science, \\ Tehran, Iran \\ ${ }^{4}$ Network of Immunity in Infection, Malignancy and Autoimmunity (NIIMA), Universal Scientific Education and Research Network \\ (USERN), Tehran, Iran \\ ${ }^{5}$ Laser Research Centre, Faculty of Health Science, University of Johannesburg, Doornfontein, Johannesburg, 2028 South Africa \\ ${ }^{6}$ Network of Immunity in Infection, Malignancy and Autoimmunity (NIIMA), Universal Scientific Education and Research Network \\ (USERN), Johannesburg, 2028 South Africa \\ ${ }^{7}$ Department of Immunology, School of Medicine, Tehran University of Medical Sciences, Tehran, Iran
}

Correspondence: N. Rezaei, Research Center for Immunodeficiencies, Children's Medical Center Hospital, 62 Qarib St, Keshavarz Blvd, Tehran 14194, Iran.: N. Rezaei

<rezaei_nima@tums.ac.ir>

Accepted for publication November 2, 2020

To cite this article: Maleki A, Khanmiri JM, Eshrat Abadi MK, Soveyzi F, Moazzami B, Hamblin MR, Rezaei N. Innate lymphoid cell subsets and their cytokines in autoimmune diseases. Eur. Cytokine Netw. 2020; 31(4): 118-128. doi: 10.1684/ecn.2020.0460

\begin{abstract}
Both the innate and adaptive arms of the immune system are involved in the development of autoimmune diseases. The main mechanism of disease is due to adaptive immune cells that are active against self-antigens. These cells can cause major damage to body tissues. Innate lymphoid cells (ILCs) are an important type of innate immune cell, whose role has been highlighted in recent years. ILCs are responsible for some of the inflammation in the pathogenesis of autoimmune diseases. In this review, we discuss the role of ILCs in the immune response, as well as their involvement in various autoimmune diseases.
\end{abstract}

Key words: innate lymphoid cells, mucosal immune response, cytokines, autoimmunity

\section{INTRODUCTION}

Innate lymphoid cells (ILCs) are a part of the innate immune system, and are tissue-resident lymphocytes that are mainly found in the mucosal membranes of the body, such as the skin and intestines [1]. These cells are also located in lymphoid tissues such as lymph nodes, as well as in adipose tissues [2,3]. ILCs are morphologically similar to $\mathrm{B}$ and $\mathrm{T}$ lymphocytes, but the recombination-activating genes (RAG) in ILCs are inactive, and they are not able to carry out the rearrangement activity needed to produce a repertoire of different immunoglobulin receptors or T-cell receptors (TCR) [4]. Similar to other lymphocytes, ILCs originate from common lymphoid progenitors (CLPs) in the fetal liver and the adult bone marrow [5]. ILCs are classified into three subgroups based on their capability to express transcription factors and cytokines, which are called ILC1, ILC2, and ILC3. ILC1 cells include natural killer (NK) cells that express T-bet and eomesodermin, while other ILC1s express only Tbet. Interferon $\gamma(\mathrm{IFN}-\gamma)$ is produced by ILC1s. ILC2s are somewhat similar to Th2 cells in that they express GATA-3 and secrete IL4, IL5, and IL13. The RARrelated orphan receptor gamma $(\mathrm{ROR} \gamma)$ is expressed by ILC3s that also synthesize IL-17A and IL-22. In a general classification, ILCs can also be divided into helper cells $\left(\mathrm{IL} \mathrm{R}_{\alpha_{+}}\right)$that include ILC1, ILC22, ILC3, and cytotoxic cells (IL7 $R_{\alpha-}$ ), including cNKs. Lymphoid tissue inducer (LTi) cells are a subtype of ILC3s that play a major role in the formation of secondary lymphoid tissues [6-9]. In addition, ILCs are key mediators in tissue stromal cell homeostasis, tissue remodeling and repair, and early immune responses to infections and inflammation [1]. Autoimmune diseases are caused by a disruption in the body's own immune tolerance mechanism, followed by an unregulated attack against the host cells. Discovering the underlying mechanisms of autoimmune diseases can be of great help in treating these disorders [10]. There are several contributing factors to autoimmune disease such as self-recognizing immune cells with or without autoantibodies. There are similarities in some of the etiologic features and pathogenesis mechanisms of different autoimmune diseases. These similarities lead to the approaches used in the treatment of these diseases also bearing some similarities [11]. One of the cell types involved in autoimmune disease is the innate lymphoid cells (ILCs). Many studies have investigated the role of these cells in the development of autoim- 
mune diseases $[12,13]$. Our objective in this review is to summarize the relationship between ILCs and diseases such as atopic dermatitis, psoriasis, systemic lupus erythematosus (SLE), multiple sclerosis, rheumatoid arthritis, irritable bowel disease (IBD), systemic sclerosis, and juvenile dermatomyositis (JDM).

\section{DEVELOPMENT AND DIFFERENTIATION OF ILC LINEAGES}

A new family of lymphoid cells discovered within the body was called ILCs, which include cytotoxic (natural killer [NK] cells) ILCs, and helper ILCs, both of which play an essential role in the body's innate immune system. ILCs are identified based on the expression of CD127 and are classified into three groups according to the transcription factors (TF) and cytokines that they produce. ILCs in Group 1 (ILC1s) express the TBX21 gene encoding T-BET, and produce gamma interferon (IFN-g) and tumor necrosis factor alpha (TNF-a), which are both $\mathrm{T}$ helper (TH) 1-associated cytokines. ILCs in Group 2 (ILC2s) express GATA-3 and related orphan receptor A (RORA) and secrete TH2-related cytokines such as IL-5 and IL-3. ILCs in Group 3 use RORC to produce IL-17 and IL-22 (TH17-related cytokines) [14-17].

Hematopoietic stem cells (HSCs) give rise to all the different blood cell progenitors, among which common lymphoid progenitors (CLPs) are precursors of all the ILC subtypes as well as B cells and T cells [18, 19]. Recently, an early ILC progenitor, also known as the common innate lymphoid progenitor (CILP), was identified as CXCR6+ cells within the $\alpha$ LP population [20]. This progenitor is dependent on the transcription factor NFIL3 (nuclear-factor, interleukin 3 regulated) basic leucine zipper [21]. After the $\alpha \mathrm{LP}$, the lineage divergence of ILC population has been shown to be the common helper-like innate lymphoid progenitor (CHIP: Id2+ Lin- IL-7Ra+ $\alpha 4 \beta 7+$ CD25- PLZF $+/-$ ) [22]. In addition, lymphoid tissue inducer (LTi) cells as well as CD4+ T-cells can be derived directly from CHILP [6]. Cells with a high level of PLZF (promyelocytic leukemia zinc finger protein) and $\alpha 4 \beta 7 / \mathrm{Lin}-/ \mathrm{IL}-$ 7Ra+/cKit+ and CXCR6- are defined as the innate lymphoid cell progenitor (ILCP) population that originates from the CHILP [19]. Recently it has been shown that both cytotoxic and helper ILCs can be derived from RORgt+ ILCPs [23]. Also one study found that peripheral blood (PB) CD117+ cells (multi-potent ILCPs) led to various ILC subsets both in vivo and in vitro, including EOMES+ NK cells. While CD117+ cells are considered to be the same as circulating ILC3s, a study showed that peripheral blood CD117+ ILCs were different from gut CD117+ ILCs (NKp44- and $\left.\mathrm{RORgt}^{-}\right)$. Circulating CD117+ ILCs express IL-1R $1^{+}$, CD45RA+, CD69- (while gut resident ILC3s are $\mathrm{CD} 9^{+}, \mathrm{IL}^{-1 \mathrm{R} 1^{-}}, \mathrm{CD} 4 \mathrm{RA}^{-}$) and do not express the proteins T-BET or EOMES. CD117+ ILCs express high levels of TFs (ID2, GATA3, TOX, and TCF7) which have been shown to be essential for murine ILC development. These results suggest that peripheral blood CD117+ ILCs differ from ILC3s and may represent an unusual ILC subset. They may be lymphoid-biased progenitors reminiscent of multipotent ILCPs carrying a TF profile with a key signature of mature ILC genes. They demonstrated that human CD117+ ILCPs developed from CD34+ HSCs in vivo. Their model for human ILCP development suggested that pluripotent $\mathrm{CD} 34^{+}$HSCs could differentiate into multipotent ILCPs (CD34- ${ }^{-} D 7^{+} \mathrm{CD} 127^{+} \mathrm{CD} 117^{+}$ CD45RA ${ }^{+}$) with the potential to become ILC1s, ILC2s, ILC3s, and EOMES ${ }^{+} \mathrm{NK}$ cells (table 1) [17].

ILCPs generate all the ILC subsets, but may be unable to produce NK cells or LTi-like subsets. A deficiency in the expression of PLZF (promyelocytic leukemia zinc finger) led to defective development of ILC1s and ILC2s, but PLZF was not necessary for development of all ILCs. Since GATA-3 is expressed in ILCPs, several models of GATA-3 deficiency have shown to be significantly associated with a decrease in ILC1s, ILC2s, and in some cases in ILC3s (but not NK cells or LTi-like cells) $[22,24,25]$. ILCs are a heterogeneous population of immune cells with two main features: A) They have a lymphoid origin derived from CLPs and are related to IL-2R $\gamma$ c signaling. B) These cells lack specific antigen receptors and therefore do not require RAG proteins for their development [16]. cNK and ILC1 cells respond to intracellular pathogens such as intracellular bacteria and viruses [26]. Both types of cells express the activating receptors of $\mathrm{cNK}$ cells, for example NK1.1 and NKp46, but often ILC1s do not have the activating and inhibitory receptors such as the Ly49 family that recognizes the MHCI molecule expressed on normal tissues [27]. ILC1s are different from cNK cells that require the transcription factor Tbet for lineage specificity [28]. Also, the PLZF transcription factor is important for the development of ILC1s, but it is not necessary for cNK cells. Finally, the GATA3 transcription factor is crucial for the development of ILC1s, although it has not been clearly determined yet whether or not this factor is important for $\mathrm{cNK}$ cell development. $\mathrm{cNK}$ cells may be directly derived from CLPs $[6,24]$. It should be noted that the requirement for NFIL-3 transcription factor for the development of ILC1s may not be needed after infections or exposure to environmental factors [29]. In addition, two separate groups of IFN-g-secreting ILC1s have been described. One group is T-BET+ cells that express high levels of CD127 and CD161 [28]. Another group called intraepithelial ILC1s are present at mucosal sites, and are $\mathrm{CD}_{103}{ }^{+}, \mathrm{NKp} 44^{+}$, and $\mathrm{CD}_{127^{-}}$[30]. Both these ILC1s react to IL-12 by producing IFN-g, but unlike NK cells, they express minimal EOMES. Human GATA- ${ }^{+}{ }^{+}$ILC2s express the chemoattractant receptor CRTh2, IL-25R, and IL33R. They also produce IL-5 and IL-13 in physiopathological situations [31, 32].

ILC2s are present in various tissues including the lungs, bone marrow, intestinal lamina propria, skin, and liver [33]. The secretion of IL-3 and IL-5 by ILC2s is considered essential for protection against helminth and nematode infections [34]. Recently, it has been found that the development of ILC2s is dependent on GATA-3, ROR $\alpha$, TCF-1, and Notch transcription factors [35]. Other factors such as vitamin 
A and food-derided metabolites play an important role in the differentiation and maturation of ILCs2 [36]. Furthermore, specific functional ILC2s require Gfi1, as shown by a lack of the Gfil gene resulting in the nonexpression of GATA3 [37].

ILC3s include ROR $\gamma$ t fetal lymphoid tissue-inducer (LTi) cells (in fetal lymphoid tissues) and CD127+, CD117+ lineage cells, which secrete IL-17A in response to IL-23. In addition a subset of ILC3s express the natural cytotoxicity receptors (NCRs) and IL-22 [38, 39]. In Group 3 ILCs (ILC3s), the retinoic acid-related orphan receptor $\gamma t(\mathrm{ROR} \gamma \mathrm{t})$ is expressed in both humans and mice, which is necessary for the development of this group. The lack of ROR $\gamma t$ in animal models resulted in impaired development of ILC3s. Subunits of ILC3s express cell surface markers, such as NKp46, NKG2D, NKp44, and CD56 [39, 40]. IL-23 and IL-1 stimulate the expression of ROR $\gamma \mathrm{t}+$ in ILC3s, and production of IL-22 and IL-17 [18, 41, 42]. ILC3s can be either NKP46+ that express a high level of CCR6, or NKP46- that express only low amounts of CCR6 $[43,44]$. Lymphoid-tissue-inducer (LTi) cells are another subset of ILCs that use the ROR $\gamma$ transcription factor to enhance the formation of secondary lymphoid tissue in the fetus [21]. Deletion of GATA3 causes loss of the PLZF+ non-LTi progenitors, but not the LTi progenitors, which express ROR $\gamma \mathrm{t}$. The GATA3 expression is always high in PLZF+ nonLTi progenitors, whereas it is low in ROR $\gamma t+\mathrm{LTi}$ progenitors. In a study by Zhong et al. they showed that non-LTi and LTi progenitors required the Id2 transcriptional regulator for their generation, but could be distinguished from each other by differential requirements for the transcription factor GATA3. Nonetheless, for the generation of functionally mature LTi cells, a low level of GATA3 expression was required [45].

\section{CYTOKINES PRODUCED BY INNATE LYMPHOID CELLS}

ILCs are divided into two main groups, identified as cytotoxic ILCs and helper ILCs, similar to $\mathrm{CD} 4^{+}$and $\mathrm{CD} 8^{+} \mathrm{T}$ cells. The helper ILC group includes 3 subgroups, whereas the cytotoxic ILC group includes natural killer (NK) cells [26]. According to previous surveys, there are at least three types of ILCs, which produce their own individual cytokines and immune responses. Each type of ILCs has its own unique transcription factor profile. To date, 4 groups of ILCs have been defined: ILC1s or NKs (group 1 ILCs), ILC2s (group 2 ILCs), ILC3s or LTi cells (group 3 ILCs), and NK17/NK1 cells (ILC4s) [46]. The interleukin-7 receptor subunit alpha (IL-7Ra) is a common characteristic along with the absence of other common cell lineage markers [47]. In another study by L. Mazzurana et al., it was found that the growth of helper ILCs depends on IL17 because they express IL-7Ra, but this finding could not be generalized to NK cells [48].

ILC2s express CD45, IL-17RB (a receptor for IL-25), IL-33R ST2, ICOS (CD278), CD127, and Thy1, although lineage-specific cell-surface markers are not expressed on the surface of this group of ILCs $[49,50]$. Innate cytokines such as IL-33 and IL-25 can be produced by ILC2s (Lin- CD127+ ILC subset) that in turn induce the production of IL-13 and IL-5 [49-51]. For example, ILC2s contribute to pro-inflammatory responses in various organs, such as the respiratory tract and nasal cavity, and can also play an important role in the defense against helminth infections such as those caused by Nippostrongylus brasiliensis, Trichuris muris, or Strongyloides venezuelensis [49,50]. Thus, the lineage negative $\mathrm{CD} 127^{+}$ILC subset appears to regulate $\mathrm{TH} 2$ cells in innate immunity. According to recent studies, endogenous TSLP or IL-7 is required

Table 1

General features of ILCs.

\begin{tabular}{|c|c|c|c|c|c|c|}
\hline & Tissue factor & Cytokines & Tissue (mice) & Phenotypic markers & Diseases & Ref \\
\hline ILC1 & $\begin{array}{l}\text { T-bet } \\
\text { Eomesodermin }\end{array}$ & IFN- $\gamma$ & $\begin{array}{l}\text { BM, Spleen, PLN, MLN, } \\
\text { LP and IE (small and large } \\
\text { intestine), white fat and liver }\end{array}$ & $\begin{array}{l}\text { - NK cells: NK1.1+, NKp46 } \\
+ \text {, CD49a-, CD49b+, } \\
\text { TRAIL-, CD127-, CD69-, } \\
\text { CXCR6-, CXCR3- } \\
\text { - ILC1: NK1.1+, NKp46+, } \\
\text { CD49a+, CD49b-, TRAIL+, } \\
\text { CD127+/-, CD69+, CXCR6+, } \\
\text { CXCR3+ }\end{array}$ & $\begin{array}{l}\text { CD } \\
\text { SLE } \\
\text { MS } \\
\text { RA } \\
\text { JDM } \\
\text { Scleroderma }\end{array}$ & $\begin{array}{l}{[6-9,12,78,106,} \\
109,113,120, \\
127,142]\end{array}$ \\
\hline ILC2 & GATA-3 & $\begin{array}{l}\text { IL4, IL5, } \\
\text { and IL13 }\end{array}$ & $\begin{array}{l}\text { Healthy skin, PLN, MLN, } \\
\text { lung, LP and IE (small in- } \\
\text { testine), LP (large intestine), } \\
\text { white fat, and liver }\end{array}$ & $\begin{array}{l}- \text { Lin-, CD127+, CD25+, } \\
\text { Sca1+, KLRG1+, ST2+ }\end{array}$ & $\begin{array}{l}\text { UC } \\
\text { AD } \\
\text { MS }\end{array}$ & $\begin{array}{l}{[6-9,86,98,113,} \\
127,142]\end{array}$ \\
\hline ILC3 & $\mathrm{ROR} \gamma$ & $\begin{array}{l}\text { IL-17A } \\
\text { and IL-22 }\end{array}$ & $\begin{array}{l}\text { Healthy skin (NCR), spleen, } \\
\text { PLN, MLN, lung, LP and } \\
\text { IE (small and large intes- } \\
\text { tine), white fat, and liver }\end{array}$ & $\begin{array}{l}\text { NKp46+ ILC3:NK1.1-, } \\
\text { NKp46-, CD127+ } \\
\text { - NKp46- ILC3:NK1.1-, } \\
\text { NKp46+, CD127+ } \\
\text { - LTi Like:NK1.1-, NKp46-, } \\
\text { CD127+, CD4+/-, CCR6+ }\end{array}$ & $\begin{array}{l}\text { Psoriasis }\left(\mathrm{NCR}^{+}\right) \\
\text {SLE } \\
\text { MS } \\
\text { RA } \\
\text { Scleroderma }\end{array}$ & $\begin{array}{l}{[6,113,} \\
120,142]\end{array}$ \\
\hline
\end{tabular}

BM: bone marrow; LP: lamina propria; PLN: peripheral lymph node; MLN: mesenteric lymph node; IE: intraepithelial ingredient; CD: Crohn's disease; UC: ulcerative colitis; AD: Atopic dermatitis; RA: Rheumatoid Arthritis; MS: Multiple sclerosis; SLE: Systemic lupus erythematosus. 
for triggering STAT5 signaling, and ILC2s use this signaling pathway for the production of IL-5 and IL13 [50]. The NF-kB and mitogen-activated protein kinase (MAPK) signaling pathways could be downregulated by IL-25 as well as by IL-1 family members. Activation of these signaling pathways will result in high levels of IL-5/IL-13 expression by ILC2s. In this regard, several unknown interactions may exist between IL2 and Th cells, which are not yet fully understood [49, 50]. Previous studies showed that IL-2 may induce ILC2 immune responses and ILC2s could have a role in the differentiation of $\mathrm{TH} 2$ cells from naive $\mathrm{T}$ cells in a manner depending on cell-to-cell contact [49]. TH2 cells are unable to produce IL-4 or IL-13 in response to IL-25 or ILC2s. This ability may be acquired after stimulation with the cognate antigen or by treatment with PMA/ionomycin [50].

ILC1s, including NK cells and noncytotoxic helper ILC1s, produce TNF $\alpha$ and INF $\gamma$ as the main cytokines after stimulation by IL12 and IL18 cytokines [46, 48]. These cells do not release cytokines related to $\mathrm{TH} 2$ or TH17 cells [46]. The T-box transcription factor 21 (Tbx21) encodes the immune cell transcription factor called T-bet that controls the growth of TH1 cells [52]. The role of T-bet is significant in both the innate and acquired immune systems because it is expressed at high levels by the ILC1 subgroup in humans [46, 52]. The EOMES gene encodes the T-box brain protein 2 (Tbr2) known as eomesodermin. Both T-bet and eomesodermin are transcription factors that are needed for the development of NK cells. In one study by Liying Guo et al., they described the prototypic form of ILCs as NK cells which express IL-7Ra (CD127) and the cytokine common gamma (gc) receptor chain which allows them to function as cytotoxic ILCs. In fact, the collaboration of two T-box transcription factors may be needed for the complete function of NK cells. Conventional NK (cNK) cells and thymic NK (tNK) cells are two distinct types of NK cells that both produce IFN $\gamma$. Depending on the amount of IFN $\gamma$. produced, they can be classified as ILC1s. They produce IFN $\gamma$ to provide help to Th1 CD4+ cells in the innate immune response [50]. CD56bright NK cells release more cytokines such as IFN $\gamma$. The characteristic features of human IFN- $\gamma$ secreting ILCs are a high expression of T-bet, moderate expression of ROR $\gamma \mathrm{t}$, and no expression of CD117 or EOMES transcription factor. The third group of ILCs is made up of lymphoid tissue inducers (LTis) and ILC3s (Lin-CD127+ ROR $\gamma \mathrm{t}+$ ) which can produce IL-17A and IL-22 in the same way as TH cells. Moreover, IL-22 is produced by NCR+ ILC3s (NK22 cells, NCR22 cells, or NKR-LTi cells) when they induce lymphoid cell populations within the gastrointestinal tract [46].

\section{ILCS IN HOMEOSTASIS AND IMMUNITY}

The majority of ILCs are found in epithelial barrier surfaces such as the mucosal surfaces, skin and lungs. The ILCs present in these surfaces play an important role in maintaining homeostasis and tissue integrity [53]. The presence of ILCs in these tissues, particularly in the intestinal lumen, is necessary for maintaining tissue integrity and encouraging regeneration after inflammatory conditions $[54,55]$. ILC precursors express the $\alpha 4 \beta 7$ integrin that interacts with the MadCAM-1 adhesion molecule that is highly expressed on the vascular endothelial surface of mucosal lymphoid tissue such as Peyer's patches. These cells also express the chemokine receptor CXCR6 that helps the cells to migrate into the intestinal tissue [56, 57]. Acquired immune cells modulate the ILC response through various mechanisms such as regulatory $\mathrm{T}$ lymphocytes (Tregs) that control the proliferation of NK cells and ILC2s by lowering IL2 [58, 59]. During the inflammatory processes caused by infections in the intestinal tract, ILC2s release amphiregulin (a ligand for the EGF receptor) in response to the IL33 that is secreted by intestinal tissue. Activation of the EGF receptor in the intestine leads to the production of extra mucin, which has an important role in protecting against invading microorganisms in the bowel [60]. The tuft cells of the intestinal tissue secrete IL 25 in response to infection by helminth species, which is activated by ILC2s, and subsequently these cells will produce IL13. These cytokines enable intestinal stem cells to differentiate to tuft cells and goblet cells, resulting in the elimination of infection in the intestinal tract $[61,62]$. In the nervous system, the neuromedin U (NMU) neurotransmitter could be released in response to helminth infection. ILC2s express receptors for NMU, which could affect IL-5, IL-13, and amphiregulin all produced by ILC2s and which play a role in tuft-and-goblet cell differentiation [63, 64]. Influenza virus infection and its associated lung inflammation is responsible for the production of IL33 by epithelial cells as a result of activation of ILC2s in the lungs [65]. IL22 is essential for defense against enteropathogenic bacteria and viruses. A high level of IL22 is produced by ILC3s, which leads to the expression of Reg3 proteins in the epithelial cells that also play a protective role against bacterial infections $[55,66]$. Activation of the STAT3 pathway by ILC3s can stimulate the production of mediators that induce proliferation of intestinal epithelial cells, and repair damaged gut cells after chemotherapy [67]. ILCs also play a role in host metabolism. ILC2s are active in fatty tissue in response to IL-33, and release methionine enkephalin peptides that affect adipocyte precursors and result in the formation of "beige" adipocytes. This process requires a large amount of energy, resulting in weight loss in the host [68]. ILC1s are activated in the adipose tissue via IL-12 leading to an increase in IFN- $\gamma$, and the production of macrophages with a more inflammatory phenotype, leading to the development of insulin resistance in the adipose tissue $[69,70]$.

\section{ILC PLASTICITY}

ILCs in humans display a significant amount of plasticity. The abundance of ILCs present at mucosal surfaces suggests that these cells should be able to easily respond to environmental triggers. This flexibility in these cells is mainly due to their capability to interact with a variety of signaling pathways. ILC1s can produce IFN- $\gamma$ and are dependent on the T-bet 
transcription factor. Cells that are similar to ILC1s could be generated from ILC2s and ILC3s under certain conditions, and these cells are also linked to the T-bet transcription factor $[28,30]$. In humans ILC3s can be converted into ILC1s by the IL12 produced by CD14+ DCs under inflammatory conditions, and go on to induce the expression of T-bet, which activates the Notch 2 signaling pathway. During this process, the expression of the ROR $\gamma$ t transcription factor disappears and the production of IL22 is lower, although it does increase the production of IFN- $\gamma$. This process is reversible by the action of IL2 and IL3 cytokines [28]. In inflammatory conditions, such as Crohn's disease or chronic obstructive pulmonary disease, ILC2s can be converted into ILC1s by the effects of IL-12 and IL-1 $\beta$ cytokines, as well as the transcription factor $\mathrm{T}$-bet. This process can be reversed under the influence of IL 4 [71, 72]. The transformation of NK cells into ILC1-like cells in mice exposed to TGF- $\beta$ and the SMAD4 transcription factor has been reported in the tumor microenvironment [73].

\section{ILC SUBSETS IN AUTOIMMUNE DISEASES}

\section{Juvenile dermatomyositis (JDM)}

Juvenile dermatomyositis (JDM) is a systemic autoimmune disease characterized by skin and muscle symptoms in children. JDM may also affect other vital organs. The incidence of the disease is 2-4 cases per million children $[74,75]$. In the pathogenesis of this disease, both the acquired immune system (B lymphocytes and $\mathrm{T}$ lymphocytes) and the innate immune system (NK cells) are involved [76]. According to the findings, the percentage of B cells and CD4 T cells in the blood of patients with JDM is increased compared to control groups, and autoantibodies can be detected in more than $60 \%$ of these patients [76, 77]. Evidence suggests that NK cells may also be involved in the pathogenesis of JDM, because studies have shown that NK cells are decreased in patients with JDM compared to controls [78]. NK cells as innate lymphocytes play an important role in antiviral and antitumor defenses through the release of cytotoxic granules and some cytokines [79]. According to some studies, NK cells can also act as immunoregulatory cells, and therefore defects in these cells may lead to autoimmune diseases [80]. In patients with JDM, increased B cells and naive CD4+ T lymphocytes, and decreased NK cells have been detected in peripheral blood compared to a control group, which could suggest a possible link between these cell types and the disease [76, 78, 81]. Alterations in the PLC $\gamma 2$ signaling pathway in NK cells leading to dysregulated PLC $\gamma 2$ signaling were detected in treatment-naive JDM patients compared to a control group, leading to a decrease in NK cell cytotoxicity in JDM [76]. Thus, impaired immune regulation may be a major factor in the development of JDM. According to the regulatory role of NK cells in the human immune system, it can be concluded that their reduction may be involved in pathogenesis of JDM.

\section{Atopic dermatitis}

Atopic dermatitis (AD) is a chronic autoimmune skin disease. A dysfunctional epithelial barrier due to mutations in the filaggrin gene and defects in the immune system are the main causes of $\operatorname{AD}[82,83]$. Filaggrin is considered one of the most important skin proteins. A filaggrin deficiency can have a significant effect on epidermal formation and function [84]. ILC2s are present in healthy skin, and recent studies have shown that ILC2s may have a significant impact on the pathogenesis of allergic conditions such as AD [85]. In one study by Mashiko et al. [86] they showed that Th2 cells and ILC2s were upregulated in the skin lesions of patients with AD. ILC2s and Th2 cells are responsible for the creation of type 2 immune responses [87]. ILC2s in the skin lesions of patients with AD expressed high levels of CD25/IL-33R/CRTH2/CD161 [88]. Prostaglandin D2 (a ligand for CRHT2) is secreted by mast cells upon activation and results in the accumulation of basophils and ILC2s at different sites within the skin $[86,89,90]$. ILC2s can be activated by TSLP, IL-25, IL33 (epithelial cytokines), IL2, IL4, IL7, IL9 (cytokines produced by Th cells), PGD2, and cysteinyl leukotriene (lipid mediators) [38, 85]. Activated ILC2s produce IL4, IL5, IL13 (type 2 cytokines), and amphiregulin that collectively could cause skin inflammation [91].

\section{Psoriasis}

Psoriasis is a chronic skin disorder characterized by the involvement of the innate and adaptive immune systems, dysfunction of keratinocytes, epidermal hyperproliferation, and the formation of erythematous and scaly plaques [92-94]. The main underlying etiology of this disorder is primarily inflammatory cytokines such as TNF- $\alpha$, IL17A, IL23, IL-22, and IFN- $\gamma$. The major sources of IL17 are the $\gamma \delta$ T cells, $\alpha \beta$ $\mathrm{T}$ cells, neutrophils, mast cells, iNKT cells, both adaptive and natural Th17 cells, Tc17 cells (a subset of CD8+ cells) and ILC3s [95]. Lande et al. showed that activation of CD4+ and/or CD8+ $\mathrm{T}$ cells against cathelicidin (an antimicrobial peptide) is observed in two-thirds of psoriatic patients, and cathelicidin is also known as the psoriasis autoantigen. The expression of cathelicidin by keratinocytes is upregulated by IL17 and subsequently results in an increased production of LL37 (another antimicrobial peptide) that is involved in inflammation. Moreover, ADAMTS-like protein 5 (ADAMTSLP5) acts as an autoantigen in psoriasis and is produced by melanocytes resulting in the overexpression of IL-17A and IFN- $\gamma$ [95-97]. One of the most important cell types involved in psoriasis are ILCs. Teunissen et al. showed that both ILC2s and NCR-ILC3s were dominant in healthy skin, whereas $\mathrm{NCR}+$ ILC3s were present in both the skin lesions and the peripheral blood of psoriatic patients. Both IL- $1 \beta$ and IL-23 were able to convert NCR-ILC3s into NCR + ILC3s in cell culture [98]. Both of these cytokines induced NCR + ILC3s and promoted IL-17A and IL22 production [99]. Similarly, Wawrzycki et al. [100] showed that IL-22 levels were higher in psoriatic patient serum and IL-22 could be considered to be a 
target for therapy. Although IL17C is a dominant cytokine in skin lesions (compared to other IL-17 family members), IL-17A is thought to be more active in the skin and plays an important role in psoriasis pathophysiology [95, 101]. IL17A affects endothelial cells, chondrocytes, fibroblasts, synovial cells, monocytes, and keratinocytes. IL17A and IL17F stimulate keratinocytes resulting in the production of cytokines, $\beta$-defensins, antimicrobial peptides, IL-8, CCL20, and CCL2, all of which can attract neutrophils, macrophages, lymphocytes, and monocytes into the lesions. All of these molecules have been found to be increased in psoriatic plaques [102]. TNF- $\alpha$ has a main role in the onset of inflammation, and in combination with IL-17, can lead to increased gene expression in keratinocytes that are involved in the development of lesions in psoriatic patients [95].

\section{Systemic sclerosis (Scleroderma)}

Systemic sclerosis (Ssc or scleroderma) is a chronic multisystem connective tissue disease defined by increased collagen production by fibroblasts, resulting in progressive fibrosis of the skin and visceral organs, with an autoimmune inflammatory background. This disease is also characterized by widespread functional and structural abnormalities in the small blood vessels of the skin [103, 104]. Despite 50 years' research on this topic, the basic pathogenesis mechanisms of Ssc are still unknown [105]. Studies have shown that immune cells, such as regulatory $\mathrm{T}$ cells (Tregs) and $\mathrm{T}$-helper 17 (Th17) cells, as well as ILCs, may be involved in the pathogenesis of Ssc [103]. A study by Florence Roan et al. found that the number of CD4+ ILC1s and NKp44 ILC3s, but not CD4- ILC1s or ILC2s, were increased in the peripheral blood of individuals with SSc, a disease characterized by fibrotic vascular pathology, and immune dysregulation. Therefore, the authors suggested that CD4+ and CD4- ILC1s may have a role in the pathogenesis of SSc [106]. Another study by Mayuka Horikawa et al. on Ssc patients showed that the frequency and absolute number of NK cells were increased in diffuse cutaneous SSc (dcSSc), whereas they were normal in limited cutaneous SSc (1cSSc). NK cells from both dcSSc and $1 \mathrm{cSSc}$ patients exhibited an activated phenotype characterized by upregulation of CD16 and CD69 and downregulation of CD62L expression. IFN- $\gamma$ production by nonstimulated NK cells from both dcSSc and lcSSc patients was higher compared to normal controls, whereas on stimulation, a reduced amount of IFN- $\gamma$ was produced. IL-5 and IL-10 production by nonstimulated NK cells and IL-6 production by stimulated NK cells were increased in dcSSc patients, but not in $1 \mathrm{cSSc}$ patients. Thus, these results suggested that altered $\mathrm{NK}$ cell function led to immunological abnormalities in Ssc [104].

\section{Systemic lupus erythematosus}

Systemic lupus erythematosus (SLE) is a chronic autoimmune disease that can involve several different organ systems [107]. Dysregulation of the innate and adaptive immune systems is known to be the main cause of SLE [108]. Recently, a possible link between ILCs and SLE has been reported. Guo et al. showed that ILC1s were the dominant type of ILCs in the peripheral blood of patients with SLE, whereas ILC2s and ILC3s were markedly reduced. This study found that in patients with renal lesions, the ratio of ILC1s/ ILCs was considerably higher than in patients without renal lesions [12]. In another study, the number of ILC1s (Lin-CD127+ CD45+ CRTH2-CD117-cells) and ILC3s (Lin-CD127+ CD45+ CRTH2-CD-117+ cells) was associated with nephritis in SLE patients, whereas the levels ILC2s were lower in these patients [109]. In an animal model, the expression of cytokines by kidney-resident ILC2s was shown to be suppressed by IFN- $\gamma$ and IL-27 which were produced by activated $\mathrm{T}$ cells in the nephritic kidneys [110].

\section{Multiple sclerosis (figure 1)}

Multiple sclerosis (MS) is another autoimmune disease that attacks the central nervous system (CNS), and is characterized by demyelination of neurons resulting in a debilitating illness $[111,112]$. Mouse models have shown that ILC1s and ILC3s play an important role in the neuronal inflammation, by increasing the proliferation of $\mathrm{T}$ cells within the CNS. By contrast, the activation of ILC2s led to decreased $\mathrm{T}$ cell responses in the $\mathrm{CNS}$ and reduced inflammation [113]. The T-bet+ ILCs contain both ILC1s and ILC3s, which stimulate the expression of matrix metalloproteinases (MMPs) and pro-inflammatory chemokines. CD4+ T lymphocytes such as TH17 cells then gain entry into the brain parenchyma. These $\mathrm{T}$ cells are responsible for the production of inflammatory cytokines within the CNS [114]. In the CNS, ILC2s can be activated by IL33 resulting in a decreased number of TH17 and TH1 cells, ultimately leading to reduced CNS inflammation in a mouse model $[115,116]$.

\section{Rheumatoid arthritis (figure 1)}

Rheumatoid arthritis (RA) is a chronic autoimmune disease that primarily affects the joints and ultimately leads to the destruction of bone and cartilage [117]. ILCs are involved in the pathogenesis of RA, and possibly other chronic inflammatory diseases, based on the known involvement of IL7 and IL7-R, and alpha-beta lymphotoxin in the development of RA disorders. Increasing the expression of IL7 causes the induction of alpha-beta-lymphotoxin in RA patients, leading to joint inflammation. In addition, increased expression of IL7-R in the synovial tissue has been observed. Therefore, administration of soluble IL7-R alpha might be considered a therapeutic approach for the management of RA [118]. In one study by Bekiaris et al. [13] they showed that a CD4+ CD3innate-like lymphoid population of ILCs was present in the peripheral blood of RA patients. ILC3 is thought to be responsible for the production of IL5 and IL7 that are overexpressed in the joints of spondyloarthritis patients [119]. Moreover, it has been shown that ILC1s and ILC3s are present in the 
synovial fluid of RA patients and are positively correlated with the clinical severity of the disease [120]. One study showed that CD3-CD56+ NKp44 + CCR6+ cells were elevated in the peripheral blood and synovial fluid of RA patients. Although CD56 is known as an NK cell marker, it is also expressed on a subset of ILC3s. These ILC3like cells secrete TNF $\alpha$ and IL-22 and stimulate the proliferation of RA fibroblast-like synoviocytes. Moreover, the frequency of ILC3-like cells in the synovial fluid of these patients was significantly associated with the 28-joint disease activity score, indicating that ILC3s played a major role in the disease development [121].

\section{Inflammatory bowel disease (figure 1)}

Inflammatory bowel diseases (IBD) are a chronic inflammatory condition and are divided into ulcerative colitis (UC) and Crohn's disease (CD). IBD is characterized by lesions in the intestinal mucosa and submucosa. Several intrinsic and extrinsic factors are believed to contribute to IBD pathogenesis including hereditary factors, environmental factors, altered gut microbiome, and inappropriate immune responses [122124]. Patients suffering from long-term IBD are at increased risk for colon cancer [125]. Studies have shown that the dysregulation of IL17/IL23 as a result of disturbances in the innate and adaptive immune responses is associated with the development of IBD. In the context of the innate immune response, ILCs play an important role in the development of this disease. Forkel et al. demonstrated that there was increased expression of ILC1s in CD as well as ILC2s in UC, which were responsible for inflammation in different parts of the gastrointestinal tract $[123,126,127]$. An alteration in the normal gut microbiome is seen in patients with IBD $[128,129]$. Increased activation of macrophages in the mucosa and submucosa is also present in these patients [130]. Microbial stimuli induce macrophages and dendritic cells to produce IL1 $\beta$, IL12, IL18, and IL23. The impact of these cytokines on the ILC3s leads to increased production of IL17 and IL22. Epithelial cells are also stimulated by IL17 to produce IL-1 $\beta$, IL-6, and TNF $\alpha$ (pro-inflammatory cytokines) as well as chemokines. The high chemokine levels can induce neutrophil migration and also cause disruption of junctional proteins and cause gut permeability $[123,131,132]$. ILC3s can produce granulocyte-macrophage colony-stimulating factor which affects myeloid cells leading to increased inflammation [133]. In the case of a vitamin A-deficient diet, IL22 production is reduced by ILC3s, which predisposes individuals to Citrobacter rodentium infection [36, 134]. ILCs also express the death domain receptor 3 (DR3). Microbial and nonmicrobial antigens increase TL1A expression in mononuclear phagocytes such as DCs and macrophages within the intestinal lamina propria. The presence of IL1 $\beta$, IL23, IL2, and the TL1A/DR3 signaling pathway resulted in ILC3 proliferation and increased IL22 levels [135].

\section{CONCLUSION}

There are similarities and differences between ILCs and $\mathrm{B} / \mathrm{T}$ lymphocytes indicating that their roles in autoimmune disease may be complex [2]. The mechanisms of some autoimmune diseases are incompletely understood, but the presence of ILCs in epithelial barrier surfaces and their role in immune responses

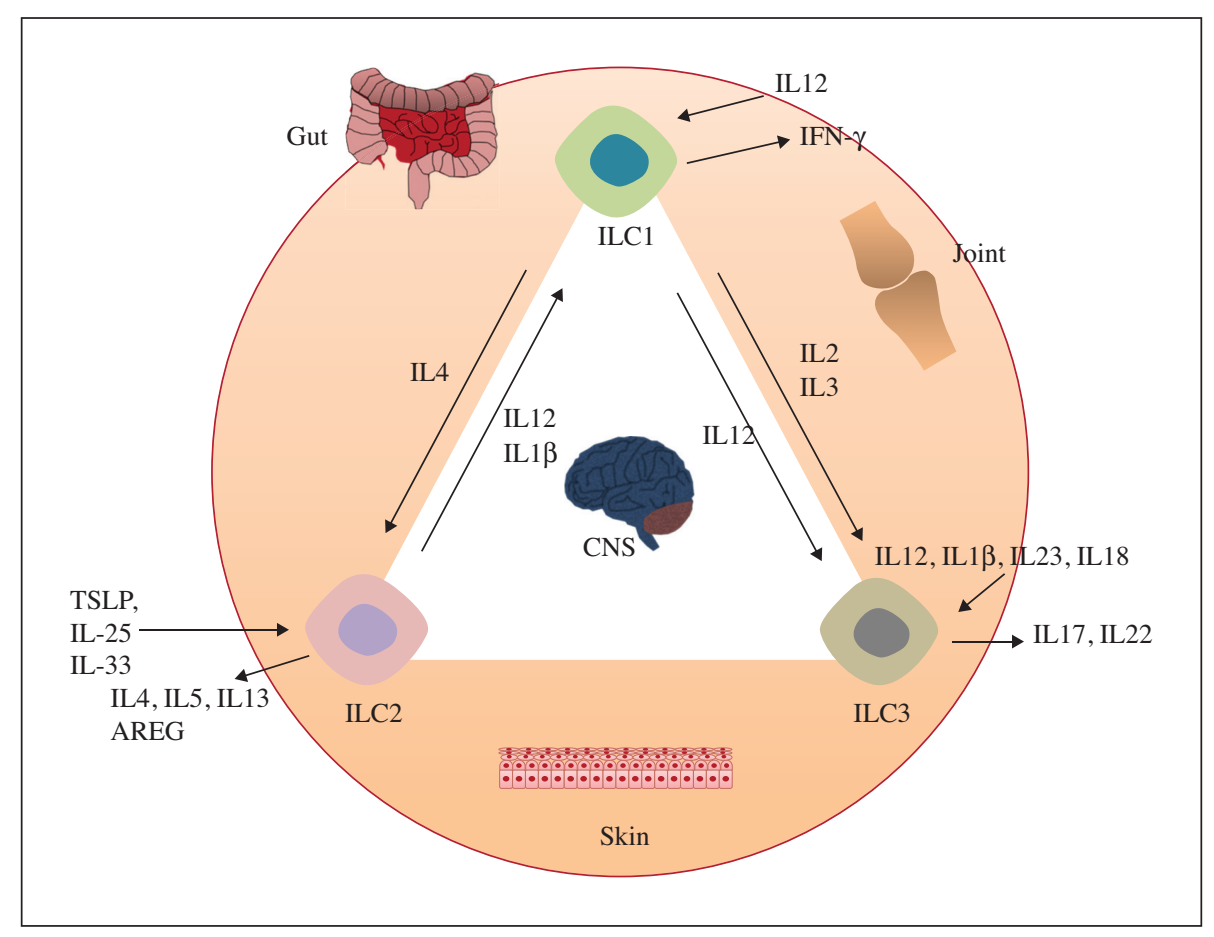

Figure 1

The role of ILC1 in Multiple sclerosis and Rheumatoid Arthritis and Crohn's disease. These cells under the influence of IL-12 can produce INFy. TSLP, IL-25, and IL-33 affect ILC2 and cause them to produce IL-4, IL-5, IL-13, and AREG. ILCs2 are seen in Multiple sclerosis and Atopic dermatitis. ILC3 plays a role in Multiple sclerosis, Psoriasis, and Rheumatoid Arthritis. ILC3 under the influence of IL-12, IL-1 $\beta$, IL-23, and IL-18 can produce IL-17 and IL-22. 
have been well demonstrated. ILCs are mostly located in secondary lymphoid tissues and are involved in regulating adaptive immune cells. This regulatory activity can be either by direct or by indirect interactions including MHCII:TCR, CD28:B7 members, TNFSF:TNFRSF members [2, 136]. Moreover, cytokine secretion, e.g. the production of IL-10 by NK cells, can suppress B and DC cells. The role of these cytokines in the pathology of autoimmune diseases has been widely studied. ILCs are associated with many autoimmune diseases, and their pathophysiology is mainly related to production of T-cell cytokines. For example, ILC1s, ILC2s, and ILC3s are all involved in IBD development, and the cytokines involved are IFN $\gamma$, perforin, granzyme, IL-13, IL-17A/F, IL-22, and IL-26. On the other hand, the role of ILCs in the anti-tumor immune response has recently gained some attention, further underlining the particular importance of these cells [137-142]. Therefore, due to the presence of ILCs in most tissues and the broad range of ILC activity in various autoimmune-related diseases, these cells could be considered a possible treatment target for the management of autoimmune disorders in the future.

Disclosure. There is no conflict of interest to disclose.

\section{REFERENCES}

1. Geremia A, Arancibia-Cárcamo CV. Innate lymphoid cells in intestinal inflammation. Front Immunol 2017; 8 : 1296.

2. Withers DR. Innate lymphoid cell regulation of adaptive immunity. Immunology 2016; 149(2):123-30.

3. Gronke K, Kofoed-Nielsen M, Diefenbach A. Innate lymphoid cells, precursors and plasticity. Immunol Lett 2016; 179 : 9-18.

4. Spits H, Artis D, Colonna M, et al. Innate lymphoid cells-a proposal for uniform nomenclature. Nat Rev Immunol 2013; 13 (2): 145 .

5. Zhong C, Zhu J. Transcriptional regulatory network for the development of innate lymphoid cells. Mediat Inflamm 2015; 2015.

6. Cortez VS, Robinette ML, Colonna M. Innate lymphoid cells: new insights into function and development. Curr Opin Immunol 2015; 32 : 71-7.

7. Jiao Y, Huntington ND, Belz GT, Seillet C. Type 1 innate lymphoid cell biology: lessons learnt from natural killer cells. Front Immunol 2016; 7 : 426.

8. Ignacio A, Breda CNS, Camara NOS. Innate lymphoid cells in tissue homeostasis and diseases. World J Hepatol 2017; 9 (23):979.

9. Huang Y, Paul WE. Inflammatory group 2 innate lymphoid cells. Int Immunol 2015; 28(1):23-8.

10. Huang C, Zhu H-X, Yao Y, et al. Immune checkpoint molecules. Possible future therapeutic implications in autoimmune diseases. J Autoimmun 2019;;102333.

11. Karagianni P, Tzioufas AG. Epigenetic perspectives on systemic autoimmune disease. J Autoimmun 2019;;102315.

12. Guo $\mathrm{C}$, Zhou M, Zhao S, et al. Innate lymphoid cell disturbance with increase in ILC1 in systemic lupus erythematosus. Clin Immunol 2019; 202 : 49-58.

13. Bekiaris V, Sedý JR, Rossetti M, et al. Human CD4+ CD3innate-like $\mathrm{T}$ cells provide a source of TNF and lymphotoxin- $\alpha \beta$ and are elevated in rheumatoid arthritis. J Immunol 2013; 191 (9):4611-8.
14. Serafini N, Vosshenrich CA, Di Santo JP. Transcriptional regulation of innate lymphoid cell fate. Nat Rev Immunol 2015; 15(7):415-28.

15. Spits H, Artis D, Colonna M, et al. Innate lymphoid cells-a proposal for uniform nomenclature. Nat Rev Immunol 2013; 13 (2):145-9.

16. Lim AI, Di Santo JP. ILC-poiesis: ensuring tissue ILC differentiation at the right place and time. Eur J Immunol 2019; 49(1):11-8.

17. Lim AI, Li Y, Lopez-Lastra S, et al. Systemic human ILC precursors provide a substrate for tissue ILC differentiation. Cell 2017; 168(6):1086-100.e10.

18. Diefenbach A, Colonna M, Koyasu S. Development, differentiation, and diversity of innate lymphoid cells. Immunity 2014; 41(3):354-65.

19. Stokic-Trtica V, Diefenbach A, Klose CS. NK cell development in times of innate lymphoid cell diversity. Front Immunol $2020 ; 11$.

20. Yu X, Wang Y, Deng M, et al. The basic leucine zipper transcription factor NFIL3 directs the development of a common innate lymphoid cell precursor. Elife 2014; 3 : e04406.

21. Seillet C, Rankin LC, Groom JR, et al. Nfil3 is required for the development of all innate lymphoid cell subsets. J Exp Med 2014; 211(9):1733-40.

22. Constantinides MG, McDonald BD, Verhoef PA, Bendelac A A committed precursor to innate lymphoid cells. Nature 2014; 508(7496):397-401.

23. Scoville SD, Mundy-Bosse BL, Zhang MH, et al. A progenitor cell expressing transcription factor ROR $\gamma \mathrm{t}$ generates all human innate lymphoid cell subsets. Immunity 2016; 44(5):1140-50.

24. Yagi R, Zhong C, Northrup DL, et al. The transcription factor GATA3 is critical for the development of all IL-7R $\alpha$ expressing innate lymphoid cells. Immunity 2014; 40(3):378-88.

25. Vosshenrich CA, García-Ojeda ME, Samson-Villéger SI, et al. A thymic pathway of mouse natural killer cell development characterized by expression of GATA-3 and CD127. Nat Immunol 2006; 7(11):1217-24.

26. Seillet C, Belz G, Huntington N. Development, Homeostasis, and Heterogeneity of NK Cells and ILC1. Curr Topics Microbiol Immunol 2015; 395.

27. Zook EC, Kee BL. Development of innate lymphoid cells. Nat Immunol 2016; 17(7):775-82.

28. Bernink JH, Peters CP, Munneke M, et al. Human type 1 innate lymphoid cells accumulate in inflamed mucosal tissues. Nat Immunol 2013; 14(3):221-9.

29. Firth MA, Madera S, Beaulieu AM, et al. Nfil3-independent lineage maintenance and antiviral response of natural killer cells. J Exp Med 2013; 210(13):2981-90.

30. Fuchs A, Vermi W, Lee JS, et al. Intraepithelial type 1 innate lymphoid cells are a unique subset of IL-12-and IL-15-responsive IFN- $\gamma$-producing cells. Immunity 2013; 38(4):769-81.

31. Mjösberg JM, Trifari S, Crellin NK, et al. Human IL-25-and IL-33-responsive type 2 innate lymphoid cells are defined by expression of CRTH2 and CD161. Nat Immunol 2011; 12 (11):1055-62.

32. Kim BS, Artis D. Group 2 innate lymphoid cells in health and disease. Cold Spring Harb Perspect Biol 2015; 7(5):a016337.

33. Hoyler T, Klose CS, Souabni A, et al. The transcription factor GATA-3 controls cell fate and maintenance of type 2 innate lymphoid cells. Immunity 2012; 37(4):634-48.

34. Koyasu S, Moro K, Tanabe M, Takeuchi T. Natural helper cells: a new player in the innate immune response against helminth infection. Adv Immunol 2010; 108 : 21-44. 
35. Yang Q, Monticelli LA, Saenz SA, et al. T cell factor 1 is required for group 2 innate lymphoid cell generation. Immunity 2013; 38(4):694-704

36. Spencer S, Wilhelm C, Yang Q, et al. Adaptation of innate lymphoid cells to a micronutrient deficiency promotes type 2 barrier immunity. Science 2014; 343(6169):432-7.

37. Spooner CJ, Lesch J, Yan D, et al. Specification of type 2 innate lymphocytes by the transcriptional determinant Gfil. Nat Immunol 2013; 14(12):1229-36.

38. Klose CS, Artis D. Innate lymphoid cells as regulators of immunity, inflammation and tissue homeostasis. Nat Immunol 2016; 17(7):765.

39. Cella M, Fuchs A, Vermi W, et al. A human natural killer cell subset provides an innate source of IL-22 for mucosal immunity. Nature 2009; 457(7230):722-5.

40. Cupedo T, Crellin NK, Papazian N, et al. Human fetal lymphoid tissue-inducer cells are interleukin 17-producing precursors to RORC+ CD127+ natural killer-like cells. Nat Immunol 2009; 10(1):66

41. McKenzie AN, Spits H, Eberl G. Innate lymphoid cells in inflammation and immunity. Immunity 2014; 41(3):366-74.

42. Cella M, Miller H, Song C. Beyond NK cells: the expanding universe of innate lymphoid cells. Front Immunol 2014; 5 : 282.

43. Van De Pavert SA, Mebius RE. New insights into the development of lymphoid tissues. Nat Rev Immunol 2010; 10 (9):664-74

44. Klose CS, Kiss EA, Schwierzeck V, et al. A T-bet gradient controls the fate and function of CCR6- ROR $\gamma \mathrm{t}+$ innate lymphoid cells. Nature 2013; 494(7436):261-5.

45. Zhong C, Zheng M, Cui K, et al. Differential Expression of the Transcription Factor GATA3 Specifies Lineage and Functions of Innate Lymphoid Cells. Immunity 2020; 52(1):83-95.e4.

46. Elemam NM, Hannawi S, Maghazachi AA. Innate Lymphoid Cells (ILCs) as Mediators of Inflammation. Release of Cytokines and Lytic Molecules. Toxins (Basel) 2017; 9(12):398.

47. Salimi M, Xue L, Jolin H, et al. Group 2 Innate Lymphoid Cells Express Functional NKp30 Receptor Inducing Type 2 Cytokine Production. J Immunol 2016; 196(1):45-54.

48. Mazzurana L, Rao A, Van Acker A, Mjösberg J. The roles for innate lymphoid cells in the human immune system. Semin Immunopathol 2018; 40(4):407-19.

49. Mirchandani AS, Besnard A-G, Yip E, et al. Type 2 innate lymphoid cells drive CD4+ Th2 cell responses. The J Immunol 2014; 192(5):2442-8

50. Guo L, Junttila IS, Paul WE. Cytokine-induced cytokine production by conventional and innate lymphoid cells. Trends Immunol 2012; 33(12):598-606

51. Fort MM, Cheung J, Yen D, et al. IL-25 induces IL-4, IL-5, and IL-13 and Th2-associated pathologies in vivo. Immunity 2001; 15(6):985-95.

52. Lazarevic V, Glimcher LH, Lord GM. T-bet: a bridge between innate and adaptive immunity. Nat Rev Immunol 2013; 13 (11):777-89

53. Nenci A, Becker C, Wullaert A, et al. Epithelial NEMO links innate immunity to chronic intestinal inflammation. Nature 2007; 446(7135):557-61.

54. Zenewicz LA, Yancopoulos GD, Valenzuela DM, Murphy AJ, Stevens S, Flavell RA. Innate and adaptive interleukin-22 protects mice from inflammatory bowel disease. Immunity 2008; 29(6):947-57.

55. Zheng Y, Valdez PA, Danilenko DM, et al. Interleukin-22 mediates early host defense against attaching and effacing bacterial pathogens. Nat Med 2008; 14(3):282-9.
56. Klose CS, Flach M, Möhle L, et al. Differentiation of type 1 ILCs from a common progenitor to all helper-like innate lymphoid cell lineages. Cell 2014; 157(2):340-56.

57. Lucas M, Schachterle W, Oberle K, Aichele P, Diefenbach A. Dendritic cells prime natural killer cells by trans-presenting interleukin 15. Immunity 2007; 26(4):503-17.

58. Hepworth MR, Fung TC, Masur SH, et al. Group 3 innate lymphoid cells mediate intestinal selection of commensal bacteria-specific CD4+ T cells. Science 2015; 348(6238):1031-5.

59. Gasteiger G, Hemmers S, Bos PD, Sun JC, Rudensky AY. IL2-dependent adaptive control of NK cell homeostasis. $J$ Exp Med 2013; 210(6):1179-87.

60. Monticelli LA, Osborne LC, Noti M, Tran SV, Zaiss DM, Artis D. IL-33 promotes an innate immune pathway of intestinal tissue protection dependent on amphiregulin-EGFR interactions. Proc Natl Acad Sci 2015; 112(34):10762-7.

61. Gerbe F, Sidot E, Smyth DJ, et al. Intestinal epithelial tuft cells initiate type 2 mucosal immunity to helminth parasites. Nature 2016; 529(7585):226-30.

62. Fallon PG, Ballantyne SJ, Mangan NE, et al. Identification of an interleukin (IL)-25-dependent cell population that provides IL-4, IL-5, and IL-13 at the onset of helminth expulsion. $J$ Exp Med 2006; 203(4):1105-16.

63. Klose CS, Mahlakõiv T, Moeller JB, et al. The neuropeptide neuromedin $\mathrm{U}$ stimulates innate lymphoid cells and type 2 inflammation. Nature 2017; 549(7671):282-6.

64. Cardoso V, Chesné J, Ribeiro H, et al. Neuronal regulation of type 2 innate lymphoid cells via neuromedin U. Nature 2017 549(7671):277-81

65. Monticelli LA, Sonnenberg GF, Abt MC, et al. Innate lymphoid cells promote lung-tissue homeostasis after infection with influenza virus. Nat Immunol 2011; 12(11):1045-54.

66. Aujla SJ, Chan YR, Zheng M, et al. IL-22 mediates mucosal host defense against Gram-negative bacterial pneumonia. Nat Med 2008; 14(3):275-81.

67. Aparicio-Domingo P, Romera-Hernandez M, Karrich JJ, et al. Type 3 innate lymphoid cells maintain intestinal epithelial stem cells after tissue damage. J Exp Med 2015; 212(11):1783-91.

68. Hams E, Armstrong ME, Barlow JL, et al. IL-25 and type 2 innate lymphoid cells induce pulmonary fibrosis. Proc Natl Acad Sci 2014; 111(1):367-72.

69. O’Sullivan TE, Rapp M, Fan X, et al. Adipose-resident group innate lymphoid cells promote obesity-associated insulin resistance. Immunity 2016; 45(2):428-41

70. Wensveen FM, Jelenčić V, Valentić S, et al. NK cells link obesity-induced adipose stress to inflammation and insulin resistance. Nat Immunol 2015; 16(4):376-85

71. Ohne Y, Silver JS, Thompson-Snipes L, et al. IL-1 is a critical regulator of group 2 innate lymphoid cell function and plasticity. Nat Immunol 2016; 17(6):646-55.

72. Bal SM, Bernink JH, Nagasawa M, et al. IL-1 $\beta$, IL-4 and IL-12 control the fate of group 2 innate lymphoid cells in human airway inflammation in the lungs. Nat Immunol 2016; 17(6):636-45.

73. Cortez VS, Ulland TK, Cervantes-Barragan L, et al. SMAD4 impedes the conversion of NK cells into ILC1-like cells by curtailing non-canonical TGF- $\beta$ signaling. Nat Immunol 2017 ; 18(9):995-1003

74. McCann L, Juggins A, Maillard S, et al. The Juvenile Dermatomyositis National Registry and Repository (UK and Ireland)-clinical characteristics of children recruited within the first $5 \mathrm{yr}$. Rheumatology 2006; 45(10):1255-60.

75. Ramanan A, Sawhney S, Murray K. Central nervous system complications in two cases of juvenile onset dermatomyositis. Rheumatology 2001; 40(11):1293-8. 
76. Throm AA, Alinger JB, Pingel JT, Daugherty AL, Pachman LM, French AR. Dysregulated NK cell PLC $\gamma 2$ signaling and activity in juvenile dermatomyositis. JCI Insight 2018; 3(22).

77. Tansley SL, Simou S, Shaddick G, et al. Autoantibodies in juvenile-onset myositis: their diagnostic value and associated clinical phenotype in a large UK cohort. J Autoimmun 2017; 84 : 55-64.

78. O'Gorman MR, Bianchi L, Zaas D, Corrochano V, Pachman LM. Decreased levels of CD54 (ICAM-1)-positive lymphocytes in the peripheral blood in untreated patients with active juvenile dermatomyositis. Clin Diagn Lab Immunol 2000; 7(4):693-7.

79. Cooper MA, Fehniger TA, Turner SC, et al. Human natural killer cells: a unique innate immunoregulatory role for the CD56bright subset. Blood 2001; 97(10):3146-51.

80. Gross CC, Schulte-Mecklenbeck A, Rünzi A, et al. Impaired NK-mediated regulation of T-cell activity in multiple sclerosis is reconstituted by IL-2 receptor modulation. Proc Natl Acad Sci 2016; 113(21):E2973-82.

81. Eisenstein DM, O'Gorman M, Pachman LM. Correlations between change in disease activity and changes in peripheral blood lymphocyte subsets in patients with juvenile dermatomyositis. J Rheumatol 1997; 24(9):1830-2.

82. Morita H, Moro K, Koyasu S. Innate lymphoid cells in allergic and nonallergic inflammation. J Allergy Clin Immunol 2016; 138(5):1253-64.

83. Weidinger S, Novak N. Atopic dermatitis. Lancet 2016; 387 (10023):1109-22.

84. Cabanillas B, Novak N. Atopic dermatitis and filaggrin. Curr Opin Immunol 2016; 42 : 1-8.

85. Cosmi L, Liotta F, Maggi L, Annunziato F. Role of type 2 innate lymphoid cells in allergic diseases. Curr Allergy Asthma Rep 2017; 17(10):66.

86. Mashiko S, Mehta H, Bissonnette R, Sarfati M. Increased frequencies of basophils, type 2 innate lymphoid cells and Th2 cells in skin of patients with atopic dermatitis but not psoriasis. J Dermatol Sci 2017; 88(2):167-74.

87. Lambrecht BN, Hammad H. The immunology of asthma. Nat Immunol 2015; 16(1):45.

88. Kim BS, Siracusa MC, Saenz SA, et al. TSLP elicits IL-33-independent innate lymphoid cell responses to promote skin inflammation. Sci Transl Med 2013; 5 (170):170ra16-ra16.

89. Xue L, Salimi M, Panse I, et al. Prostaglandin D2 activates group 2 innate lymphoid cells through chemoattractant receptor-homologous molecule expressed on TH2 cells. $J$ Allergy Clin Immunol 2014; 133(4):1184-94.e7.

90. Chang JE, Doherty TA, Baum R, Broide D. Prostaglandin D2 regulates human type 2 innate lymphoid cell chemotaxis. $J$ Allergy Clin Immunol 2014; 133(3):899-901.e3.

91. Ebbo M, Crinier A, Vély F, Vivier E. Innate lymphoid cells: major players in inflammatory diseases. Nat Rev Immunol 2017; 17(11):665.

92. Schön MP. Adaptive and Innate Immunity in Psoriasis and Other Inflammatory Disorders. Front Immunol 2019; 10.

93. Huang T-H, Lin C-F, Alalaiwe A, Yang S-C, Fang J-Y. Apoptotic or antiproliferative activity of natural products against keratinocytes for the treatment of psoriasis. Int $J \mathrm{Mol}$ Sci 2019; 20(10):2558.

94. Benhadou F, Mintoff D, Del Marmol V. Psoriasis: keratinocytes or immune cells - Which is the trigger? Dermatology 2019; 235(2):91-100.

95. Blauvelt A, Chiricozzi A. The immunologic role of IL-17 in psoriasis and psoriatic arthritis pathogenesis. Clin Rev Allergy Immunol 2018; 55(3):379-90.
96. Lande R, Botti E, Jandus C, et al. The antimicrobial peptide LL37 is a T-cell autoantigen in psoriasis. Nat Commun 2014; 5 : 5621 .

97. Arakawa A, Siewert K, Stöhr J, et al. Melanocyte antigen triggers autoimmunity in human psoriasis. J Exp Med 2015; 212(13):2203-12

98. Teunissen MB, Munneke JM, Bernink JH, et al. Composition of innate lymphoid cell subsets in the human skin: enrichment of NCR + ILC3 in lesional skin and blood of psoriasis patients. J Invest Dermatol 2014; 134(9):2351-60.

99. Xiong T, Turner J-E, editors. Innate lymphoid cells in autoimmunity and chronic inflammatory diseases. Sem Immunopathol 2018.

100. Wawrzycki B, Pietrzak A, Grywalska E, Krasowska D, Chodorowska G, Rolinski J. Interleukin-22 and its correlation with disease activity in plaque psoriasis. Arch Immunol Ther Exp (Warsz) 2019; 67(2):103-8.

101. Johnston A, Fritz Y, Dawes SM, et al. Keratinocyte overexpression of IL-17C promotes psoriasiform skin inflammation. J Immunol 2013; 190(5):2252-62.

102. Martin D, Townw J, Krikorian G, Klekotka P, Gudjonsson J, Krueger J. The Emerging Role of Interleukin-17 in the Pathogenesis of Psoriasis: Preclinical and Clinical Findings. J Invest Dermatol: 17-26.

103. Varga J, Wigley FM. Scleroderma-systemic sclerosis. Clin Immunol 2019;;743-55e1.

104. Horikawa M, Hasegawa M, Komura K, et al. Abnormal natural killer cell function in systemic sclerosis: altered cytokine production and defective killing activity. J Invest Dermatol 2005; 125(4):Error: FPage (731) is higher than LPage (7)!.

105. Greenblatt MB, Aliprantis AO. The immune pathogenesis of scleroderma: context is everything. Curr Rheumatol Rep 2013; 15(1):297.

106. Roan F, Stoklasek TA, Whalen E, et al. CD4+ group 1 innate lymphoid cells (ILC) form a functionally distinct ILC subset that is increased in systemic sclerosis. J Immunol 2016; 196(5): Error: FPage (2051) is higher than LPage (62)!

107. Deafen D, Escalante A, Weinrib L, et al. A revised estimate of twin concordance in systemic lupus erythematosus. Arthritis Rheum 1992; 35(3):Error: FPage (311) is higher than LPage $(8)$ !.

108. Grammatikos AP, Tsokos GC. Immunodeficiency and autoimmunity: lessons from systemic lupus erythematosus. Trends $\mathrm{Mo}$ Med 2012; 18(2):Error: FPage (101) is higher than LPage (8)!.

109. Hou M, Liu S. Innate lymphoid cells are increased in systemic lupus erythematosus. Clin Exp Rheumatol 2019; 37(4):Error: FPage (676) is higher than LPage (9)!.

110. Düster M, Becker M, Gnirck AC, Wunderlich M, Panzer U, Turner JE. T cell-derived IFN- $\gamma$ downregulates protective group 2 innate lymphoid cells in murine lupus erythematosus. Eur J Immunol 2018; 48(8):Error: FPage (1364) is higher than LPage (75)!.

111. Kearney H, Altmann DR, Samson RS, et al. Cervical cord lesion load is associated with disability independently from atrophy in MS. Neurology 2015; 84(4):Error: FPage (367) is higher than LPage (73)!.

112. Frischer JM, Bramow S, Dal-Bianco A, et al. The relation between inflammation and neurodegeneration in multiple sclerosis brains. Brain 2009; 132(5):Error: FPage (1175) is higher than LPage (89)!.

113. Russi AE, Walker-Caulfield ME, Ebel ME, Brown MA Cutting edge: c-Kit signaling differentially regulates type 2 innate lymphoid cell accumulation and susceptibility to central nervous system demyelination in male and female SJL mice. $J$ 
Immunol 2015; 194(12):Error: FPage (5609) is higher than LPage (13)!.

114. Kwong B, Rua R, Gao Y, et al. T-bet-dependent NKp46+ innate lymphoid cells regulate the onset of $\mathrm{T} \mathrm{H} 17$-induced neuroinflammation. Nat Immunol 2017; 18(10):1117.

115. Jiang HR, Milovanović M, Allan D, et al. IL-33 attenuates EAE by suppressing IL-17 and IFN- $\gamma$ production and inducing alternatively activated macrophages. Eur J Immunol 2012; 42 (7):Error: FPage (1804) is higher than LPage (14)!.

116. Milovanovic M, Volarevic V, Ljujic B, et al. Deletion of IL-33R (ST2) abrogates resistance to EAE in BALB/C mice by enhancing polarization of APC to inflammatory phenotype. PLoS One 2012; 7(9):e45225.

117. Yazdani R, Sharifi M, Shirvan AS, Azizi G, GanjalikhaniHakemi M. Characteristics of innate lymphoid cells (ILCs) and their role in immunological disorders (an update). Cell Immunol 2015; 298(1-2):66-76.

118. Hartgring SA, van Roon JA, Wijk MWV, et al. Elevated expression of interleukin-7 receptor in inflamed joints mediates interleukin-7-induced immune activation in rheumatoid arthritis. Arthritis Rheum 2009; 60(9):Error: FPage (2595) is higher than LPage (605)!

119. Cuthbert R, Fragkakis E, Dunsmuir R, et al. Human enthesis group 3 innate lymphoid cells. Arthritis Rheumatol 2017; 69(9): Error: FPage (1816) is higher than LPage (22)!.

120. Koo J, Kim S, Jung WJ, et al. Increased lymphocyte infiltration in rheumatoid arthritis is correlated with an increase in LTi-like cells in synovial fluid. Immune Netw 2013; 13(6):Error: FPage (240) is higher than LPage (8)!

121. Ren J, Feng Z, Lv Z, Chen X, Li J. Natural killer-22 cells in the synovial fluid of patients with rheumatoid arthritis are an innate source of interleukin 22 and tumor necrosis factor- $\alpha . J$ Rheumatol 2011; 38(10):Error: FPage (2112) is higher than LPage (8)!.

122. Segal JP, Mullish BH, Quraishi MN, et al. The application of omics techniques to understand the role of the gut microbiota in inflammatory bowel disease. Ther Adv Gastroenterol 2019; $12: 1756284818822250$

123. Zeng B, Shi S, Ashworth G, Dong C, Liu J, Xing F. ILC3 function as a double-edged sword in inflammatory bowel diseases. Cell Death Dis 2019; 10(4):315.

124. Axelrad JE, Lichtiger S, Yajnik V. Inflammatory bowel disease and cancer: The role of inflammation, immunosuppression, and cancer treatment. World J Gastroenterol 2016; 22(20): 4794.

125. Beaugerie L, Itzkowitz SH. Cancers complicating inflammatory bowel disease. $N$ Engl J Med 2015; 372(15):Error: FPage (1441) is higher than LPage (52)!.

126. Moschen AR, Tilg H, Raine T. IL-12, IL-23 and IL-17 in IBD: immunobiology and therapeutic targeting. Nat Rev Gastroenterol Hepatol 2019; 16(3):85-96.

127. Forkel M, van Tol S, Höög C, Michaëlsson J, Almer S, Mjösberg J. Distinct Alterations in the Composition of Mucosal Innate Lymphoid Cells in Newly Diagnosed and
Established Crohn's Disease and Ulcerative Colitis. J Crohns Colitis 2018; 13(1):67-78.

128. Michail S, Durbin M, Turner D, et al. Alterations in the gut microbiome of children with severe ulcerative colitis. Inflamm Bowel Dis 2012; 18(10):Error: FPage (1799) is higher than LPage (808)!

129. Rajca S, Grondin V, Louis E, et al. Alterations in the intestinal microbiome (dysbiosis) as a predictor of relapse after infliximab withdrawal in Crohn's disease. Inflamm Bowel Dis 2014; 20(6):Error: FPage (978) is higher than LPage (86)!.

130. Lissner D, Schumann M, Batra A, et al. Monocyte and M1 macrophage-induced barrier defect contributes to chronic intestinal inflammation in IBD. Inflamm Bowel Dis 2015; 21(6): Error: FPage (1297) is higher than LPage (305)!.

131. Longman RS, Diehl GE, Victorio DA, et al. CX3CR1+ mononuclear phagocytes support colitis-associated innate lymphoid cell production of IL-22. J Exp Med 2014; 211(8): Error: FPage (1571) is higher than LPage (83)!

132. Fournier B, Parkos C. The role of neutrophils during intestinal inflammation. Mucosal Immunol 2012; 5(4):354.

133. Pearson C, Thornton EE, McKenzie B, et al. ILC3 GM-CSF production and mobilisation orchestrate acute intestinal inflammation. Elife 2016; 5 : e10066.

134. Vivier E, Artis D, Colonna M, et al. Innate lymphoid cells: 10 years on. Cell 2018; 174(5):Error: FPage (1054) is higher than LPage (66)!.

135. Valatas V, Kolios G, Bamias G. TL1A (TNFSF15) and DR3 (TNFRSF25): a co-stimulatory system of cytokines with diverse functions in gut mucosal immunity. Front Immunol 2019; 10 .

136. Nenci A, Becker C, Wullaert A, et al. Epithelial NEMO links innate immunity to chronic intestinal inflammation. Nature 2007; 446(7135):557.

137. Koh J, Kim HY, Lee Y, et al. IL-23-producing human lung cancer cells promote tumor growth via conversion of innate lymphoid cell 1 (ILC1) into ILC3. Clin Cancer Res 2019: clincanres 3458.2018.

138. Barrow AD, Colonna M. Exploiting NK cell surveillance pathways for cancer therapy. Cancers 2019; 11(1):55

139. Vulpis E, Soriani A, Cerboni C, Santoni A, Zingoni A. Cancer Exosomes as Conveyors of Stress-Induced Molecules: New Players in the Modulation of NK Cell Response. Int $J$ Mol Sci 2019; 20(3):611.

140. Wu C-H, Li J, Li L, et al. Extracellular vesicles derived from natural killer cells use multiple cytotoxic proteins and killing mechanisms to target cancer cells. J Extracell Vesicles 2019; 8 (1): 1588538 .

141. Li S, Yang D, Peng T, Wu Y, Tian Z, Ni B. Innate lymphoid cell-derived cytokines in autoimmune diseases. $J$ Autoimmun 2017; $83: 62-72$

142. Kim CH, Hashimoto-Hill S, Kim M. Migration and tissue tropism of innate lymphoid cells. Trends Immunol 2016; 37 (1):68-79. 